\title{
Performance Measurement for Construction CALS Application of Ubiquitous Technology Using the Process Chart Method
}

\author{
Tae Hak Kim, Seong Yun Jeong \\ ICT Convergence and Integration Research Division, \\ Korea Institute of Construction Technology, Goyang-Si, Republic of Korea \\ Email: kimth@re.kr
}

Received September 2013

\begin{abstract}
From the industrial field that spearheaded IT created new added values and markets, and with the fusion of the traditional industry composition, researches are actively being conducted to steer advancement. The application possibility of the high-tech IT technique in the construction industry is increasing. Therefore, when a technique similar to this ubiquitous technique is applied to the Construction CALS system, the effectiveness of the business transaction is augmented, and a business that could reduce the various needed expenses is predicted. In this research, the exhibition features developed and detailed the features of the Construction CALS system to measure the effectiveness of the development so as to prove the validity of the Process Chart using techniques with detailed exhibition features, and will want to conduct quantitative performance measurement.
\end{abstract}

Keywords: Construction CALS; Ubiquitous; Process Chart Method; Quantitative Performance Measurement

\section{Introduction}

In recent years, the national infrastructure business of the construction sector has been calling for the upgrading of construction informatization through the convergence and combination of sophisticated IT application technologies. In the construction field, the HR management and logistics management sectors are researching on the application of the RFID technology to actual work, along with the pilot application of the technology. In connection with such changes, in road/river project management, to resolve the problems with the users' manual inputting of construction field data, such as data input delay, inaccuracy and absence, a need to develop methods of using the ubiquitous technology and automatically inputting data is emerging.

Thus, the existing construction CALS system must be improved. In recent years, the functions of the construction CALS system are being improved and upgraded by using sophisticated technologies, gathering information in real time, achieving automatic connection, removing unnecessary processes, and changing approval processes, so as to enhance work efficiency, reduce manpower and shorten construction periods, and consequently cut various costs, including labor cost.

From this viewpoint, to continue develop the construction CALS system, it should be upgraded in line with environmental changes, and the performance of the upgraded functions must be analyzed.

Even though the construction of the CALS system is upgraded in a timely manner, the performance of its upgraded function should be analyzed quantitatively and verified to prove its effects.

Thus, to measure and prove the effects of the developed pilot functions and detailed functions, this study quantitatively measured the time and cost results, used the process chart technique, and offered a method of analyzing the performance of pilot functions and detailed functions.

To measure the performance of the developed construction CALS system, the performance of the system function in using its sensor network, inspecting facilities and automatically inputting/gathering onsite data was measured.

\section{Preliminary Discussion}

\section{Outline of the Construction CALS System}

CALS is an integrated informatization strategy designed to enable the client, the constructor and other related parties to exchange and share via the Internet the information created in the entire process of construction project planning and design, construction execution, and maintenance. It is based on the Construction Technology Management Act-Article 15-2 (the construction of integrated information systems for supporting construction work). The project began in 1998, and systems and construction information standards have since been developed. The fourth master plan (2013-2017) has been devised and is now being implemented.

The developed systems are the Construction Project Management System, the Facility Maintenance Management System, the Land Compensation System, the Construction Permit System and the Construction CALS Portal System (Figure 1).

\section{Discussion of Existing Researches}

To propose a method of measuring the performance of the construction CALS system, existing studies on the system were 
analyzed and compared with this study.

In recent years, to maximize the effects of the construction CALS system and to operate the system more efficiently, diverse attempts are being made and approaches taken such as the construction of the system (Yun Hoe-su, 2006) and onsite verification, it was found.

Also, to survey the quality level of the system and the satisfaction with it, evaluation models were developed (Jeong In-su, 2008).

In addition, Kim Jin-uk (2009) offered a performance system concept design for performance management and measurement, but the actual measurement system was not constructed. Thus, the effects of the CALS system improvement and application could not be verified. However, this study offers a method of analyzing the quantitative performance of the upgraded construction CALS system functions.

\section{Analysis of the Construction CALS System Function Performance}

\section{Performance Measurement Method}

To compare and analyze the current and prospective processes of the CALS system and to measure the performance of its pilot and detailed functions, the process chart technique was used.

The process chart technique is a useful method of schematizing the work details and the flow of materials, equipment and manpower into simple symbols (Table 1), and to analyze opportunities for saving time, effort and resources. As such, the technique was very useful for measuring such performance herein.

\section{Facility Inspection Work Pilot Function}

Pilot function of inspecting facilities

- To ensure inspection efficiency by measuring the natural frequency of the bridge with the sensor node

- To secure information on absences and safety

- To upgrade the bridge management function through realtime monitoring (The hardware configuration is outlined in Figure 2.)

To measure the effects of the system's pilot function of inspecting facilities using the process chart and the sensor network, the performance was measured. Regarding the process before and after the application of the pilot function that targets facility inspection using the sensor network, Table 2 shows the result of the survey and analysis of the performance using the

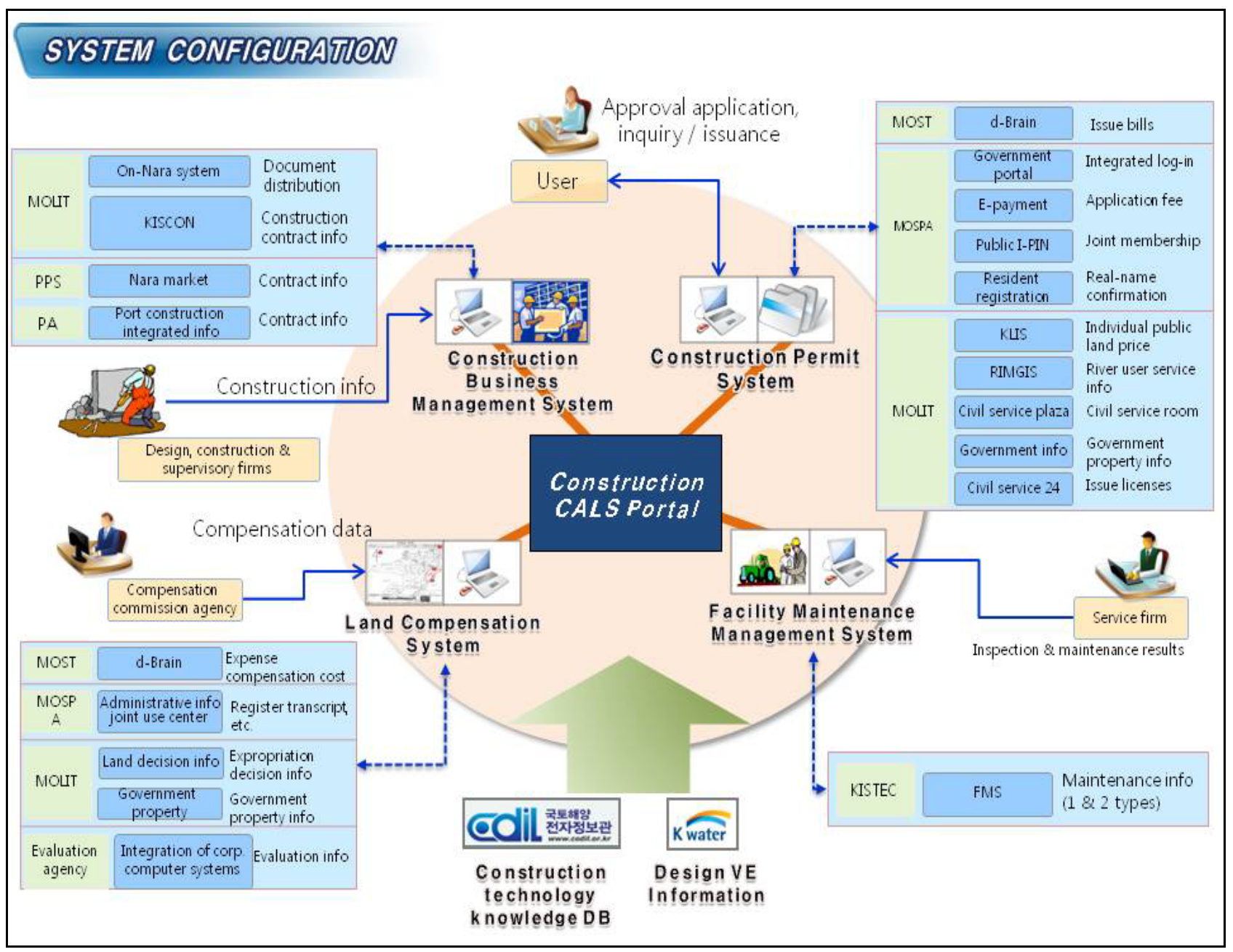

Figure 1.

Configuration of the construction CALS system. 
Table 1.

ASME (American Society of Mechanical Engineers) process symbols.

\begin{tabular}{cll}
\hline Symbol & Name & \multicolumn{1}{c}{ Meaning } \\
\hline$\square$ & Work & Behavior or displacement that accompanies changes \\
$\square$ & Move & Movement of humans or objects \\
$\square$ & Store & Long-term storage of information, materials, etc. \\
\hline
\end{tabular}

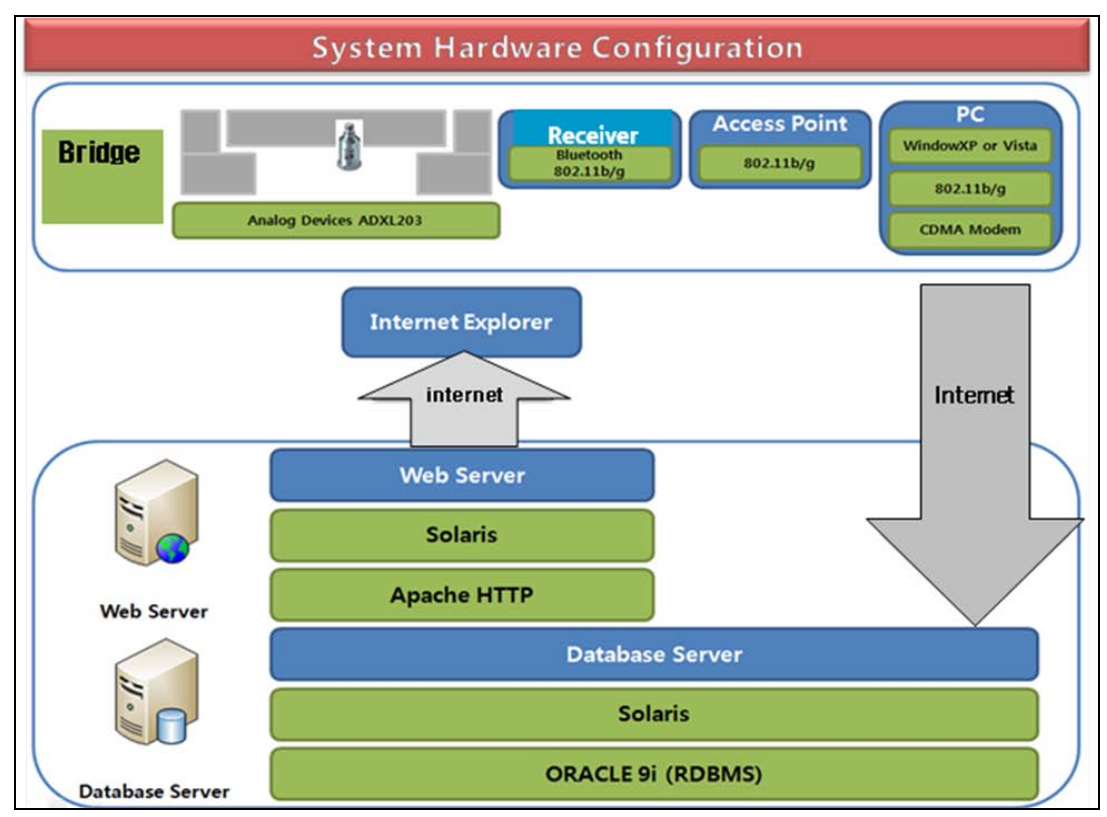

Figure 2.

Configuration of the pilot function hardware for inspecting facilities.

Table 2.

Current and prospective pilot functions of facility inspection using the sensor network based on the process chart analysis.

\begin{tabular}{|c|c|c|c|c|c|}
\hline \multicolumn{6}{|c|}{ (Unit: minute) } \\
\hline \multirow{2}{*}{$\begin{array}{c}\text { Chart } \\
\text { Symbol }\end{array}$} & \multicolumn{2}{|c|}{ As-is Process Model } & \multicolumn{2}{|c|}{ To-be Process Model } & \multirow{2}{*}{ Effect } \\
\hline & Process & Work Time & Process & Work Time & \\
\hline & Prior survey & 240 & Prior survey (web) & 30 & 210 \\
\hline$\Rightarrow$ & Move to the field & 60 & Move to the field & 60 & 0 \\
\hline & Onsite survey (Appearance survey) & 30 & Onsite survey (Appearance survey) & 30 & 0 \\
\hline$\Longrightarrow$ & Move to the office & 60 & Move to the office & 60 & 0 \\
\hline & Review and analyze the survey results & 30 & Review and analyze the survey results & 30 & 0 \\
\hline 0 & Write the report & 60 & Write the report & 60 & 0 \\
\hline$\nabla$ & Approve and store & & Approve and & & \\
\hline Total & 480 & & 270 & & 210 \\
\hline
\end{tabular}

process chart technique.

As shown in Table 2, the current facility inspection work and the inspection process were classified into four stages based on one bridge and took 360 minutes. Moreover, the prospective work and the inspection process of the developed pilot function had four stages but took 150 minutes, thus reducing the time by 210 minutes compared with the current process.

The staff's onsite visual check and review/analysis report writing took the same amount of time; but with the prospective process, the time required for identifying onsite problems before performing onsite work and for gathering related data was drastically reduced.

\section{Onsite Data Automatic Input/Gathering Functions}

The onsite-data automatic input/gathering function had the hardware configuration shown in Figure 3 to:

- Secure manage information in real time and achieve JIT 
- Check the transport time between the factory and the field and secure quality

- Automate the gathering of invoice information; and Analyze the gathered data and devise an optimal transport plan

Using the process chart technique, Table $\mathbf{3}$ shows the results of the survey/analysis of the process with the application of the onsite-data automatic input/gathering functions, based on an onsite survey and interviews with the working staffers (the survey targeted remicon work, for which the remicon, ascon and soil function is expected to be used the most), and the process before the application of the detailed functions.

As shown in Table 3, the current remicon management work and the inspection process were classified into seven stages based on one vehicle's one-time work, and took 150 minutes. Moreover, the prospective work and inspection process with the application of the developed functions had six stages, one stage less than the current process, and took 47 minutes, 103 minutes less than the current process.

\section{Conclusion}

To examine the effects of the developed pilot functions and detailed functions, the process chart technique was used and quantitatively analyzed. The findings revealed that the work time was reduced.

Specifically, in the pilot function of inspecting facilities using the sensor network, the inspection time was reduced by

Table 3.

Current and prospective onsite-data automatic input/gathering function based on the process chart analysis.

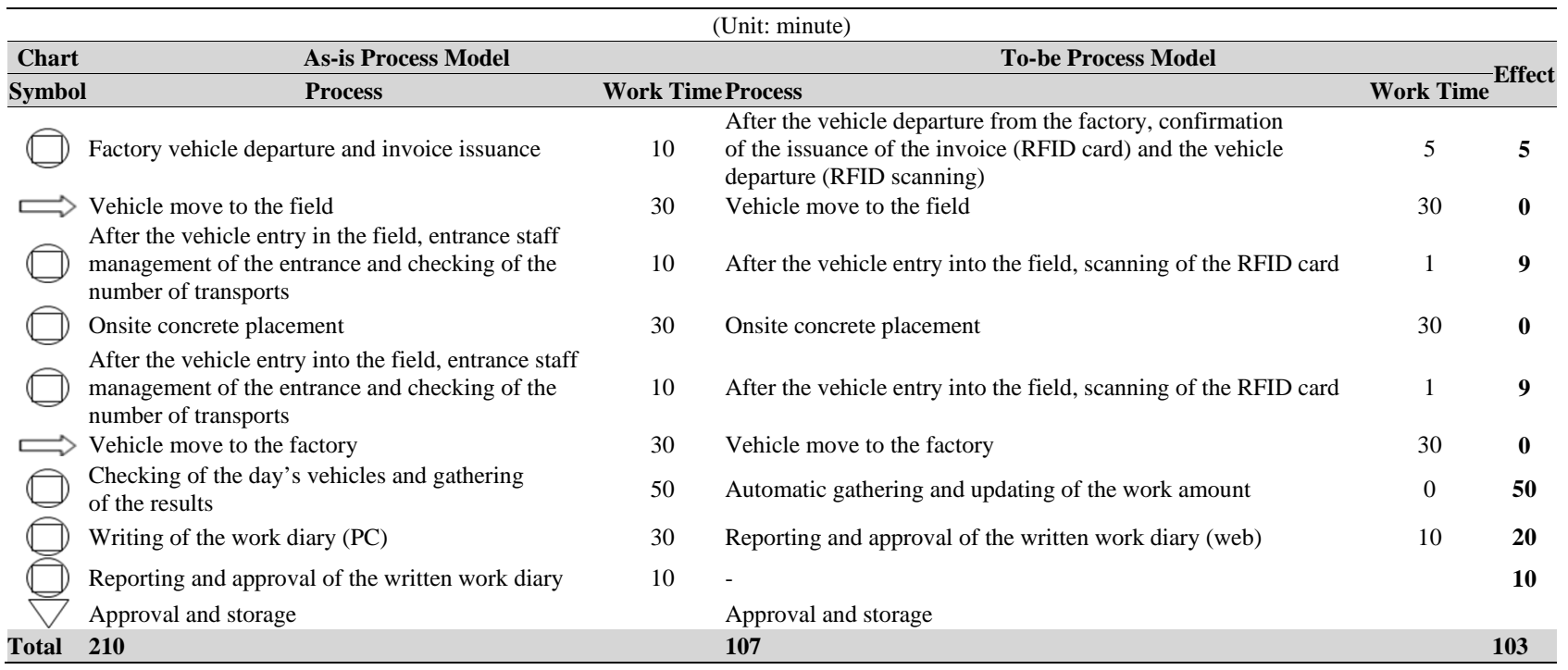

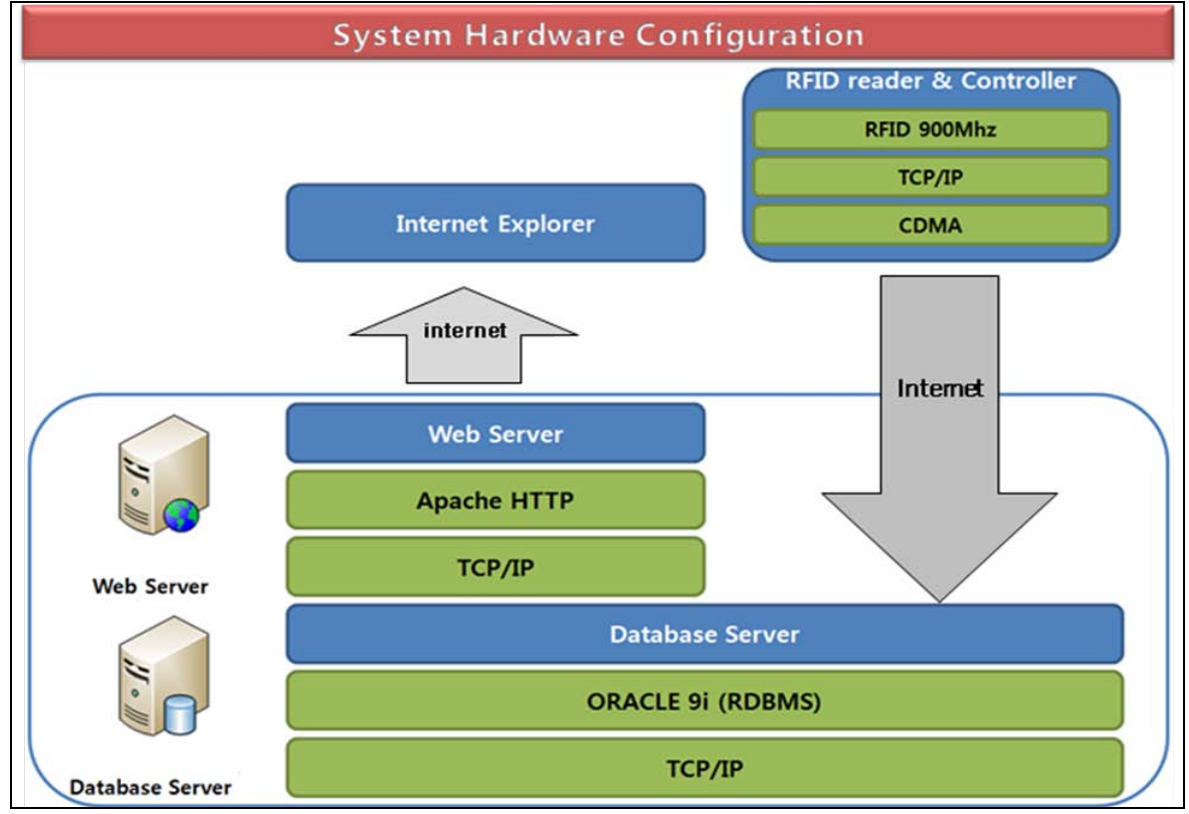

Figure 3.

Configuration of the onsite-data automatic input/gathering function hardware. 
$60 \%$ compared with the existing inspection process; and in the onsite-data automatic input/gathering function, the time was reduced by $70 \%$ compared with the existing work process.

The process chart technique that was proposed herein for quantitative performance measurement can be used to analyze the performance of other functions of the established construction CALS system to devise strategies for upgrading the construction CALS system and to come up with a method of measuring the system performance.

\section{Acknowledgements}

This study was conducted as part of the [13. Operation and Technical Improvement of the Construction CALS System (II)] by the Ministry of Land, Infrastructure and Transport.

\section{REFERENCES}

Jeong, I.-S. (2008). Analysis of the quality level of the construction CALS system. Seoul: Architectural Institute of Korea.

Kim, C.-H. (2005). USN concept and analysis of recent trends. Seoul: Korea Institute of Information and Communication.

Kim, J.-U. (2009). Design of the construction CALS performance management system. Seoul: Korean Institute of Information Technology.

Yun, H.-S. (2006). A study on the design direction of the construction CALS portal system. Seoul: Architectural Institute of Korea. 